\title{
Swiss Science Concentrates
}

A CHIMIA Column

\section{Gram-scale Synthesis of Two-dimensional Polymer Crystals and their Structure Analysis by X-ray Diffraction}

M. J. Kory, M. Wörle, T. Weber, P. Payamyar, S. W. van de Poll, J. Dshemuchadse, N. Trapp, and A. D. Schlüter*, Nat. Chem. 2014, 6, 779. ETH Zurich.

Two-dimensional polymers have fascinated chemists long before the discovery of graphene. Their controlled synthesis has however remained elusive. Schlüter and coworkers report on the synthesis of a twodimensional polymer based on polymerization within a crystal of a photoreactive monomer, followed by a delimination step. This elegant design strategy will allow to systematically investigate structure-property relationships for this emergent class of twodimensional materials.

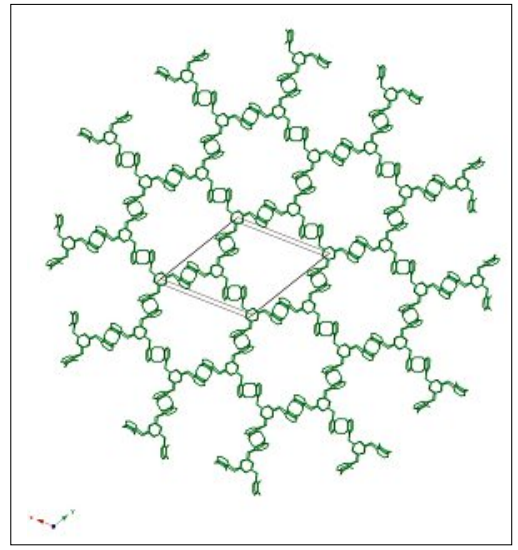

\section{Direct Synthesis of Formic Acid from Carbon Dioxide by Hydrogenation in Acidic Media}

S. Moret, P. J. Dyson, and G. Laurenczy*, Nat. Commun. 2014, doi:10.1038/ncomms5017. EPFL Lausanne.

In times of climate change, carbon capturing has become an attractive means to reduce the rise of $\mathrm{CO}_{2}$ levels. Besides the storage of $\mathrm{CO}_{2}$ in porous rock formations, its conversion to high value chemicals, including formic acid, is an attractive alternative. Laurenczy and coworkers present a catalyst for the direct hydrogenation of $\mathrm{CO}_{2}$ to formic acid in unprecedentedly high concentrations (up to $1.9 \mathrm{M}$ in DMSO). The $\mathrm{Ru}(\mathrm{II})$ catalyst system bearing watersoluble phosphine ligands can be recycled several times without loss of activity. Most importantly, it does not require the presence of base.

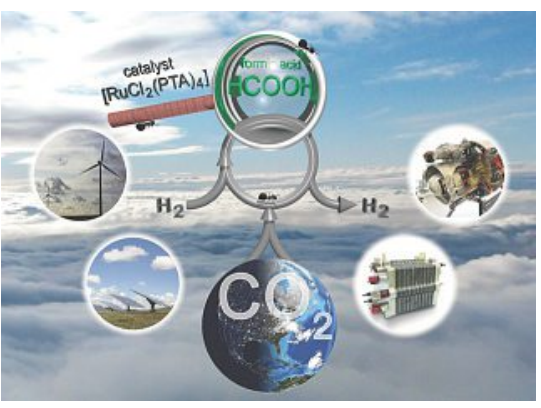

Anion- $\pi$ and Cation- $\pi$ Interactions on the Same Surface

K. Fujisawa, C. Beuchat, M. Humbert-Droz, A. Wilson, T. A. Wesolowski, J. Mareda, N. Sakai, and S. Matile*, Angew. Chem. Int. Ed. 2014, 53, 11266. University of Geneva.

The mechanism of cell-penetration of certain peptides is still under debate. One theory proposes anionic- $\pi$ and cationic$\pi$ interactions on the same surface of biological moieties, especially for guanidinium-rich peptides. Matile and co-workers addressed this question relying on both computational- and synthetic investigations. For this purpose, they enforced 'ionpair- $\pi$ ' interactions (carboxylate-guanidinium moiety) on push-pull chromophores. An intramolecular Stark effect, resulting from ionpair interactions, of $+43 \mathrm{~nm}$ was observed in $\mathrm{CCl}_{4}$ which is in near agreement with the calculated value. Protonation of the carboxylate reversibly weakens or cancels the red shifts. These results provide elegant theoretical and experimental support that anion- $\pi$ and cation- $\pi$ interactions can occur on the same surface.

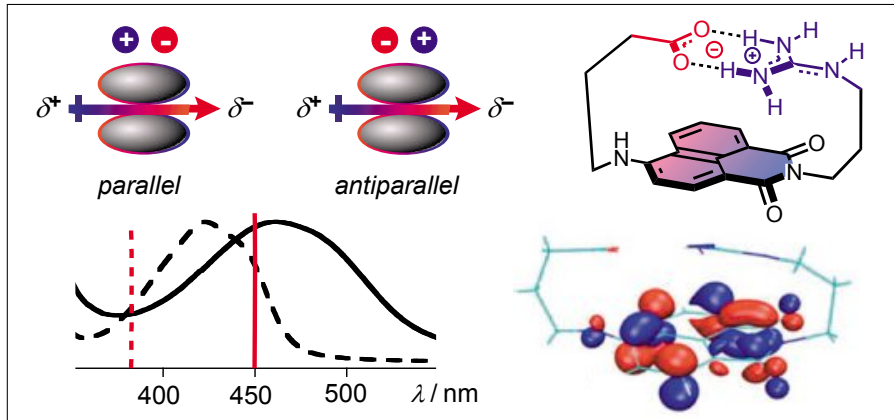

\section{An Octadentate Bifunctional Chelating Agent for the Development of Stable Zirconium-89 Based Molecular Imaging Probes}

M. Patra, A. Bauman, C. Mari, C. A. Fischer, O. Blacque, D. Häussinger, and G. Gasser*, T. L. Mindt*, Chem. Commun. 2014, 50, 11523. Universities of Zurich and Basel.

Molecular imaging agents based on the radionuclide zirconium-89 $\left({ }^{89} \mathrm{Zr}\right)$ hold great promise as novel radiotracers in nuclear medicine using high-resolution positron emission tomography. However, insufficient in vivo stability of currently used radiometal complexes employing hexadentate chelators is a safety concern in view of clinical applications. Now a consortium of researchers of the Universities of Basel and Zurich report the facile synthesis and evaluation of the first octadentate bifunctional chelating agent for the development of ${ }^{89} \mathrm{Zr}$-labelled (bio)conjugates with remarkably improved stability profiles.

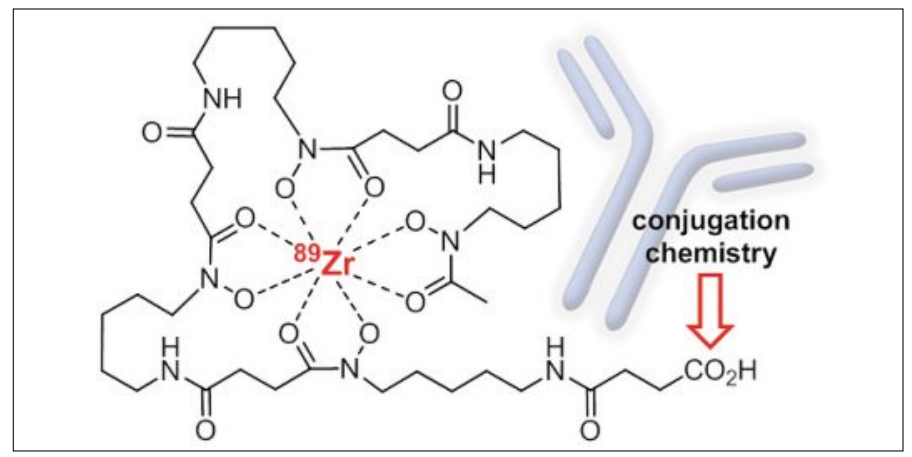

Prepared by Mariana Spulber, Valentin Köhler, Raphael Reuter, Paolo Tosatti and Thomas R. Ward 